\title{
EL MODELO DIDÁCTICO DE LA FORMACIÓN DE UN CUADRO QUÍMICO DEL MUNDO EN LOS ESTUDIANTES. UNA VÍA PARA EL CAMBIO CONCEPTUAL
}

\author{
FERRO FERNÁNDEZ, V.R. y GONZÁLEZ-JONTE CRUZ, R.H. \\ Instituto Superior Pedagógico. Pinar del Río. 20200 Cuba.
}

\section{SUMMARY}

In this paper we develop a general definition of the study of Chemistry that responds to the need to form in the students a chemical picture of the world in accordance with the didactic model developed by the authors to systematize the contents in Chemistry teaching. The concept of chemical reaction is defined in a general way, pursuing the central aim of the didactic idea, and also in an operational way, in search of a practical guide to study the chemical bond. The concepts of bond, bonding energy, and others are given a systematic treatment within these ideas.

\section{INTRODUCCIÓN}

Un profundo y complejo cambio conceptual (Hewson 1992) y metodológico (Segura 1991) se verifica en la enseñanza de las ciencias -en todos los niveles del sistema de enseñanza- y en la formación de profesores. Hewson (1992) asegura que el cambio conceptual se está popularizando, para referirse a un fenomeno que gana las comprensiones de estudiantes y profesores de diversas partes del mundo y que encuentra ya realización concreta en un esfuerzo por transformar la enseñanza de las ciencias y la formación de profesores.

El cambio conceptual responde, entre otras cosas, a la necesidad que enfrenta la escuela de hacer corresponder la preparación que brinda a los estudiantes con el nivel de desarrollo científico-tecnológico y cultural que ha logrado la humanidad. En los tiempos en que la ciencia y la técnica han revolucionado toda la estructura material, espiritual y cognoscitiva de la sociedad, la escuela está obligada a un cambio radical đe índole conceptual (¿qué enseñar?) y metodológico (¿cómo enseñar?) que modifique correspondientemente la estructura cognos. citiva del alumno.

El cambio ha sido elaborado en sus fundamentos, aspectos generales y se han desarrollado las ideas principales para su realización (Hewson 1981, 1984, Carey 1985, Hashewh 1986, Nussbaum 1986, Gunstone 1992). Ade- más, ya se cumplen con éxito experiencias pedagógicas concretas de cambio conceptual en la enseñanza de las ciencias y en la formación del personal docente en diferentes países (Hewson 1982, 1990, Lablanca 1991. Camino 1993, Pérez-Landazábal 1993).

Este último momento -ia realización concreta del cambio-presenta una especial complejidad por la diversidad cualitativa de sistemas y planes educacionales, programas y disciplinas de estudio, a través de las cuales se realiza la enseñanza de las ciencias y la formación de profesores en las diferentes partes del mundo; y hace imposible el planteamiento de soluciones generales para dicbo proceso (Hewson 1992). Tobín (1992, citado por Hewson 1992) insiste en que el cambio conceptual debe considerarse en un contexto sociocultural concreto).

El análisis precedente conduce a la comprensión de que el Iogro final del cambio conceptual exige la afiliación, el compromiso y el esfuerzo de mucho especialistas en muy diversos lugares y condiciones, de manera que las soluciones que se encuentren al problema serán también muy diferentes.

Los autores de este artículo consideran que el cambio conceptual y metodológico en química debe estar acompañado por el rescate de la integtidad conceptual de la 
propia disciplina, que se vio fraccionada en el período metafísico de su desarrollo (Sanderson 1960, Kedro 1967). Al inicio de los años sesenta, Sanderson adelantó un período de síntesis-integración en la evolución de las ciencias químicas modernas: «[...] la química, que es, $\sin$ dudas, la ciencia de mayor unidad, está a punto de dividirse por su amplitud y desarrollo, en multitud de fragmentos que difícilmente reflejen su procedencia común y unidad fundamental. Es posible que algún día se precisen quimicos generales, cuya especialidad con* sista en conservar la unidad intelectual de esta ciencia $[\ldots] \%$.

El modelo didáctico de la formación de un cuadro químico del mundo (CQM) en Ios estudiantes, planteado de manera independiente por estos autores (Ferro, González-Jonte y González 1990, 1991, Ferro y GonzálezJonte 1992), en la actividad docente y metodológica del Departamento de Química del Instituto Superior Pedagógico de Pinar del Río, coincide en su génesis y formu lación con la esencia y los fundamentos del modelo del cambio conceptual.

En el presente trabajo se explican las generalidades del modelo didáctico de la formación de un CQM en los estudiantes y se establecen los puntos de contacto con el modelo del cambio conceptual.

\section{EL MODELO DIDÁCTICO DE LA FOR- MACION DE UNCQMEN LOS ESTUDIANTES}

\subsection{Generalidades del modelo}

El perfeccionamiento del proceso docente (en la ense* nanza de las ciencias) responde, entre otras cosas, a la necesidad -referida en la introducción-de poner correspondencia entre el nivel del desarrollo científico-tecnológico del mundo contemporáneo y el estado de la instrucción escolar. El vínculo anterior puede formali* zarse como la relación entre el nivel de las ciencias y el de las disciplinas docentes correspondientes (asignaturas), que tiene dos expresiones fundamentales: la conceptual y la metodológica.

La tabla I precisa el contenido de la relación cienciaasignatura en sus dos expresiones fundamentales.

Cualquier esfuerzo cierto por un cambio conceptual en la escuela debe proponer soluciones a una de estas relaciones o a ambas simultáneamente. La que hace el modelo didáctico de la formación de un CQM en los Estudiantes «engloba» ambas expresiones y se puede resumir en dos ideas básicas.

a) Ante la imposibilidad de agotar en el tiempo de instrucción (limitado y no creciente) todo el volumen conceptual (creciente a ritmos vertiginosos) que genera el progreso científico contemporáneo, la alternativa escogida supone desarrollar los contenidos en torno a unos pocos conceptos -denominados aquí núcleos concep- tuales-que resumen lo esencial y más general del pensamiento químico moderno.

Tabla I

Contenido de la relación entre las ciencias y las disciplinas de enseñanza correspondientes.

\begin{tabular}{|c|c|}
\hline RELACIÓN & CONTENIDO \\
\hline Conceptual & $\begin{array}{l}\text { Entre los conocimientos cientificos y } \\
\text { los contenidos de enseñanza de las } \\
\text { disciplinas docentes cortespondientes. }\end{array}$ \\
\hline Metodologica & $\begin{array}{l}\text { Entre los métodos de investigación } \\
\text { de las ciencias y los métodos que se } \\
\text { utilizan cn la explicación de los } \\
\text { conteaidos de enseñanza cn la escuela } \\
\text { (métodos de enseñanza). }\end{array}$ \\
\hline
\end{tabular}

Los núcleos conceptuales constituyen los elementos primarios de la estructura conceptual en la enseñanza y el aprendizaje de la química. Ellos estructuran y sistematizan el material de estudio según la relevancia y el significado de los diferentes conceptos en el sistema cognoscitivo de la disciplina científica y docente.

La organización del proceso docente a partir de núcleos conceptuales ha tenido otros defensores (Colombo de Cudmani y Fontdevilla 1990) y con propósitos semejantes de sistematización de la actividad de enseñanza y aprendizaje del electromagnetismo tanto en el ciclo de enseñanza media como universitaria básica.

b) Aproximar las prácticas metodológicas de la investigación científica y la enseñanza.

Durante mucho tiempo la escuela ha desarrollado su propia metodología para el tratamiento de los contenidos de enser̃anza, bastante al margen -por lo menos formalmente- de los métodos de la investigación científica. El problema de los métodos de enseñanza se ha constituido una dirección prioritaria de la investigación didáctica en busca de formas particulares para la realización de la instrucción.

Los tiempos del cambio conceptual y metodológico plantean la necesidad y abren la perspectiva del acercamiento de la lógica de las disciplinas de la enseñanza a la lógica de la ciencia misma.

En el modelo didáctico de la formación de un CQM en los estudiantes esto se realiza dando una proyección metodológica al sistema de conocimientos (organizados en torno a los núcleos conceptuales) de manera que los estudiantes puedan transitar de las formulaciones generales de los núcleos conceptuales a los conocimientos particulares y, más aún, enfrentar de manera independiente y con opciones de éxito aquellos conocimientos que por razones de tiempo o complejidad no se incluyen en los currículos estudiantiles. 
En materia curricular este hecho tiene una consecuencia inmediata: la inclusión de los métodos de investigación de las ciencias químicas en el sistema de núcleos conceptuales de la carrera; proceder éste obligado si se tiene en cuenta que la metodología de una ciencia no se adquiere espontáneamente, sino que es necesario aprenderla.

Como base del sistema de núcleos conceptuales fue adoptado el objeto de estudio de la química, que transita desde el concepto de movimiento químico de la materia (de valor generalizador en los cursos de química) hasta el de reacción química (de significación operacional en la organización y explicación de los contenidos).

En el esquema 1 se resumen las relaciones fundamentales que verticalizan el modelo.

\subsection{La enseñanza y el aprendizaje de la química según el modelo didáctico de la formación de un CQM en los estudiantes}

En la práctica del proceso de enseñanza-aprendizaje según el modelo que se điscute, los núcleos conceptuales son punto de partida y retorno de la actividad cognoscitiva. El proceso transcurre como un tránsito complejo sistemático y bidireccional entre lo general (núcleos conceptuaies) y lo singular (conocimientos químicos individuales).

\section{Esquema 1}

Estructura del modelo didáctico del cuadro químico del mundo.
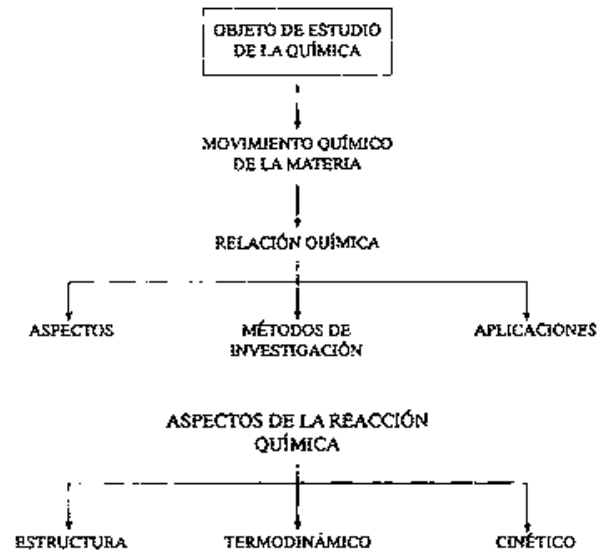

- LO CUALITATINO DE LA REACCGON QUTAGACA

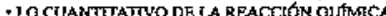
- LAS FORMAS DE REALIZACION DEZ, CAMBBOO QUIMTCO

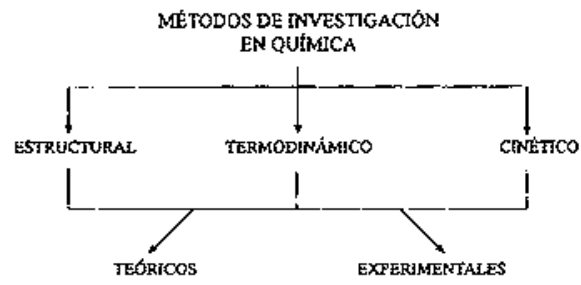

Así se parte de unos pocos conceptos o ideas básicas y se enriquecen, completan y consolidan con la contribución de todos los contenidos de la carrera, cuidando siempre el aspecto de la continuidad teórica y el devenir sistemático en la presentación de los nuevos contenidos y en la profundización de los primeros. Los nuevos contenidos deben presentarse alrededor de los primeros, de manera que vayan conformando un sistema creciente de contocimientos, tanto en su aspecto cuantitativo como cualitativo.

Se trata de partir de lo general, ir a lo particular con su guía teórica y metodológica y retornar a un nivel general (cualitativamente distinto), asimilada la experiencia de lo particular, para reelaborar el sistema inicial de conocimientos y conformar así una nueva cualidad cognoscitiva (Esquema 2).

Esquema 2

Dinámica del proceso docente según el modelo didáctico de la formación del cuadro químico del mundo.

Lo general 2

Lo general 1

(núcleos conceptuales)

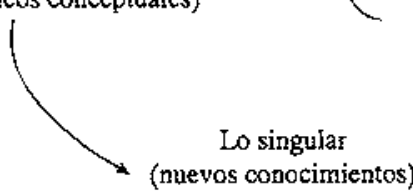

$\left(\begin{array}{c}\text { núcleos conceptuales } \\ \cdot \text { enriquecidos } \\ \cdot \text { superados }\end{array}\right)$

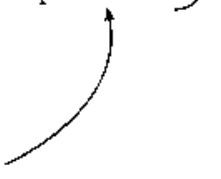

A continuación se desarrolla un ejemplo que concreta to explicado en el pártafo anterior.

\section{Ejemplo 1}

Sobre la enseñanza y el aprendizaje de un contenido nuevo en química según el modelo didáctico de la formación de un CQM en los estudiantes.

Sea el núcleo conceptual la reacción química, y considérense como nuevos conocimientos las reacciones de adición en hidrocarburos.

Desde el inicio mismo de la carrera, et estudiante debe tener una definición y comprensión general de la reacción química, que postetiormente evoluciona hacia un estadio operacional con el estudio de las reacciones típicas del mundo inorgánico (precipitación, formación de complejos, oxidación-reducción, etc.), destacánđóse su naturaleza ełectrónica. Los ejemplos que se discuten en inorgánica (aceptando su tradicional precedencia respecto a la orgánica) deben enriquecer la comprensión de dicho proceso y asi el estudiante tendrá una definictón como la siguiente:

Logeneral 1: La reacción química es un proceso [...] que transcurte con reordenamiento[...] electrónico por transferencia, compartimiento o cesión de electrones.

Donde la idea de «[...] que transcurre con reordenamiento en el estado de distribución de los electrones [...] " representa un nivel de gencralidad en que están incluidos como casos particulares los que correspon. den a las reacciones de precipitación, formación de complejos, oxidación-reducción, etc. 
- Constituye un esfuerzo simultáneo de significación conceptual y metodológica. Aqquí las expresiones conceptuales y metodológicas del cambio se funden en una manifestación didáctica única (epígrafe 1.1).

- Sus principios (epígrafe 1.1) y la dinámica de su acción (epígrafe 1.2) son válidos, tanto para la enseñanza como para el aprendizaje. En el primero de los casos constituye una vía para la organización curricular, la realización y el tratamiento de los contenidos. A los estudiantes, por su parte, les pertrecha teórica y metodológicamente para enfrentar la docencia y el trabajo individual en la incorporación de nuevos contenidos, en la sistematización de los ya apropiados, etc. Se trata đe una conceptualización teórica y metodológica que integra en una praxis única las acciones de enseñar y aprender.

De esta manera la actividad del estudiante-aprendizajeadquiere una independencia relativa de la profesoral -enseñanza-. La labor del profesor se dirige fundamentalmente a la orientación general de los estudiantes y al tratamiento de cuestiones específicas que surgen en el proceso, convirtiéndose en un elemento que lo facilita y que lo estimula. El estudiante dotado con la visión general de la ciencia que estudia puede identificar nuevos problemas y plantear soluciones para ellos.

- Es, en lo fundamental, un procedimiento constructivista (Magoon 1977) por la preocupación que observa en la construcción del sistema de contenidos del estudiante. Ve el aprendizaje como extensión e intercambio respecto al estado cognoscitivo anterior del alumno (Ejemplo 1), como superación y rescate - a la vez- de los conocimientos anteriores, como una interacción entre las conceptuaciones que el alumno posee y la nueva información que incorpora. En este arreglo didáctico para garantizar la permanencia de lo aprendido se busca que la nueva estructura tenga el mismo grado de coherencia interna y sea elaborada de acuerdo a la experiencia anterior del alumno (Posner 1982).

Un momento crítico en el logro de este propósito es -para las condiciones en que se desarrolla el modelo-el

\section{REFERENCIAS BIBLIOGRÁFICAS}

CAMINO, N., 1993. Ideas previas y cambio conceptual: un estudio con maestros de primaria sobre día y noche, estaciones y fases. Poster presentado en el IV Congreso Internacional sobre Investigación en la Didactica de las Ciencias y las Matemáticas. Barcelona.

CAREY, S., 1985. Conceptual change in childhood (1 $1^{\mathrm{a}}$ ed.). (Mit Press: Cambridge, MA).

COLOMBO DE CUDMANI, L. y FONTDEVILA, P.A., 1990. Concepciones previas en el aprendizaje significativo del electromagnetismo, Enseñanza de las Ciencias, 8(3), pp. $215-222$. tránsito del nivel preuniversitario al universitario. Los estudiantes provienen de la media con dispares niveles teóricos y una deficiente comprensión de la química (Ferro Fernández 1989). La solución dada a este problema consiste en la «superación» activa y participación de toda su ecología química-conceptual y la «instauración» de una definición general del objeto de estudio de la química con las propiedades ya discutidas (epígrafe 1.3). Este proceso supone (como todo el posterior) el reemplazamiento de las concepciones erróneas por otras correctas.

- Es consustancial con un aprendizaje problemático y está estrechamente ligado al desarrollo del pensamiento lógico y a las habilidades creativas en los estudiantes. EI esquema de su realización (Esquema 2) es propicio y seguro para el planteamiento de nuevas tareas y la búsqueda de soluciones no convencionales y creativas a las mismas.

\section{PRECISIONES FINALES}

Se han explicado los fundamentos de un modelo didáctico dirigido a la sistematización de los contenidos de enseñanza en la formación de profesores de química. El modelo didáctico de la formación de un $\mathrm{CQM}$ en los estudiantes, por sus planteamientos metodológicos y epistemológicos y si forma de realizarse, puede ser definido como un modelo de cambio conceptual.

\section{NOTAS}

${ }^{1}$ Se refiere a la transformación de una sustancia o varias en otra ut otras diferentes.

${ }^{2}$ Se trata de la estequometría del proceso.
FERRO FERNÁNDEZ, V.R., 1989. Resultados no publicados. Instituto Superior Pedagógico. Pinar del Río.

FERRO FERNÁNDEZ, V.R., 1990. Sobre la necesidad y la forma de sistematizar los contenidos de la enseñanza en la formación de profesores de química. Ponencia presentada en la Reunion Metodológica del Departamento de Quimica. Instituto Superior Pedagógico. Pinar del Río.

FERRO FERNÁNDEZ, V.R., 1991. El objeto de estudio de la química y su significación teórico-conceptual y metodológica en la formación de un cuadro químico del mundo en los estudiantes. Conferencia leída en el Departamento de Quimica del Instituto Superior Pedogógico. Pinar del Rfo. 
FERRO FERNÁNDEZ, V.R., GONZÁLEZ-JONTE, R.H. y GONZÁLEZ ORTEGA, A.M. 1992. La formación de un cuadro químico del mundo en los estudiantes como modelo para la sistematización de los contenidos químicos. II Coloquio Internacional sobre la Enseñanza Superior de la Química en Lenguas Internacionales de Origen Latino. Málaga.

FERRO FERNÁNDEZ, V.R. y GONZÁLEZ-JONTE, R.H., 1992. La formación de un cuadro químico del mundo en los estudiantes como modelo para la sistematización de los contenidos químicos, Studia Chemica. Sección Didáctica, 17 , pp. $127-136$

GUNSTONE, R.F. y NORTHFIELD, J., 1992. «Conceptual change in teacher education: the centrality of metacognition". Ponencia presentada en el Annual Meeting of the American Education Research Association. San Francisco, CA.

HASHEWH, M.Z., 1986. Towards an explanation of conceptual change, European Journal of Science Education, 3(4), pp. 383-396.

HEWSON, P.W., 1982. A case study of conceptual change in special relativity: the influence or prior knowledge in learning, European Journal of Science Education, 4(1), pp. 61-78.

HEWSON, P.W. y HEWSON, M.G. A'B, 1984. The role of conceptual conflict in conceptual change and the design of instruction, Instructional Science, 13(1), pp. 1-13.

HEWSON, P.W., 1990. La enseñanza de «fuerza y movimiento» como cambio conceptual, Enseñanza de las Ciencias, $8(2)$, pp. 157-172.

HEWSON, P.W. 1992. El cambio conceptual en la enseñanza de las ciencias y la formación de profesores. Ponencia presentada en el encuentro «Investigación y Desarrollo del Currículo en la Enseñanza de las Ciencias. Madrid.
KEDROV, B.M., 1976. Clasificación de las Ciencias. Tomo I. (Progreso: Moscú).

LABLANCA, R., 1991. Una estrategia encaminada a producir el cambio conceptual, Enseñanza de las Ciencias, 9(2), pp. 207-208.

MAGOON, A.J., 1977. Constructivist approaches in educational research, Review of Educational Research, 47 (4), pp. 651-693.

NUSSBAUM, J., 1989. Classroom conceptual change: philosophical perspectives, International Journal of Science Education, 11, pp. 530-540.

PARERA LÓPEZ, J.J., 1992. Modelo electrostático-colisional para el proceso cuántico elemental $\mathrm{H}+\mathrm{H} \rightarrow \mathrm{H}_{2}^{+}$. I. Teoría. Revista Cubana de Química. (En prensa).

PÉREZ-LANDAZABAL, M.C., GARCÍA-GALLO, J. y MORENO, J.M., 1993. «Cambio conceptuaI obtenido mediante unidades didácticas experimentales con ordenador: calor y temperatura". Póster presentado en el IV Congreso Internacional sobre Investigación en la Didactica de las Ciencias y las Matemáticas. Barcelona.

POSNER, G.J., STRIKE, K.A., HEWSON, P.W. y GERTZONG, W.A., 1982. Accomodation of a scientific conception: Toward a theory of conceptual change, Science Education, 66(2), pp. 211-227.

SANDERSON, R.T., 1960. Periodicidad química. (Aguilar: Madrid).

SEGURA, D., 1991. Una premisa para el cambio conceptual: el cambio metodologico, Enseñanza de las Ciencias, 9(2), pp. $175-180$.

SHULKIN, G.B., 1987. Química para todos. (Conocimiento: Moscú). 
